\title{
Epidemiological Analysis of Rabies in Central China from 2013 to 2018
}

\author{
Li Cai ${ }^{1,2}$ \\ Lixian Wang ${ }^{3}$ \\ Xuhua Guan ${ }^{4}$ \\ Lei Wang ${ }^{4}$ \\ Xinyi $\mathrm{Hu}^{5}$ \\ Yang $\mathrm{Wu}^{4}$ \\ Yeqing Tong ${ }^{4}$ \\ Peigang Wang' \\ 'School of Health Sciences, Wuhan \\ University, Wuhan, 43007I, People's \\ Republic of China; ${ }^{2}$ Wuhan Center for \\ Disease Control and Prevention, Wuhan, \\ 430015, People's Republic of China; \\ ${ }^{3}$ Xiaonan Center for Disease Control \\ and Prevention, Xiaogan, 432100, \\ People's Republic of China; ${ }^{4}$ Hubei \\ Center for Disease Control and \\ Prevention, Wuhan, Hubei, 430079, \\ People's Republic of China; ${ }^{5}$ Global Study \\ Institute, University of Geneva, Geneva, \\ Switzerland
}

\begin{abstract}
Objective: The study aimed to timely grasp the epidemiologic status of rabies in Central China from 2013 to 2018 and provide scientific evidence for the implementation of follow-up prevention and control measures.

Methods: We initiated a retrospective observational and descriptive study of bite-related injuries data and rabies disease data in Hubei province from 2013 to 2018, managed by the Center for Disease Control and Prevention.

Results: A total of 2,028,691 individuals were exposed to bites from 2013 to 2018, of which 221 were diagnosed with rabies and deceased. Among those cases, the incubation periods of rabies varied from 3 days to 18,406 days, which has been shown to be statistically associated with where the infected person was exposed and whether the wound care has been conducted.

Conclusion: Epidemiological studies have shown that from 2013 to 2018, the current situation of rabies in Central China is still severe. The case fatality rate keeps virtually $100 \%$. The rural population is still the most vulnerable group to rabies, characterized by a high exposure ratio and low treatment rate as well as poor vaccination compliance. Hoewever, larger populations are warranted to validate our findings.
\end{abstract}

Keywords: rabies, epidemiology, China

\section{Introduction}

Rabies, a kind of zoonoses caused by the rabies virus, is a fatal neurological disease associated with replication in the central nervous system (CNS). The case fatality rate of rabies is virtually $100 \%$. There is no effective therapeutic drug so far. In addition to that, rabies vaccination has been recognized as the most effective measure to prevent and control the disease. Due to the high fatality rate, the highly variable incubation period, and prognosis, it is urgent to update accurate data on animal bite exposure and estimate the overall incidence of animal bite across the country. ${ }^{1-6}$

China is one of the countries with a large number of rabies cases in the world. Notably, Hubei Province, with a population of nearly 60 million, is a typical representative of the economy, politics, culture, and geography in China. The high incidence rate and improper case management made rabies a great challenge, especially for rural China. To timely grasp epidemic characteristics of rabies, the current study aimed to assess the prevalence of rabies in Hubei and the incubation period as well as understand the association of rabies prevention and control in Central China. By providing scientific reference, those findings can establish a stepping stone to optimize rabies prevention and control measures. 


\section{Study Objects and Methods Study Objects}

From 2013 to 2018, 2,028,691 cases have been recorded with a history of being bitten, of which 221 diagnosed cases were further investigated face-to-face by the rabies case questionnaire formulated by the Chinese Center for Disease Control and Prevention. The questionnaire contained information on the socio-demographic characteristics of rabies, injured time, onset disease time, treatment, vaccination status. Informed consents were obtained from all participants. A parent or legal guardian provided informed consent for participants under the age of 18 . This study was conducted in accordance with the Declaration of Helsinki.

\section{Definition of Rabies Cases}

The study adopts the WHO criteria for defining a standard case of rabies: ${ }^{7}$ Clinical cases are cases with acute neurological syndrome (such as encephalitis), mainly for functional stimulated type (eg, mania) rabies or paralysis syndrome type (such as paralysis) rabies, without intensive care support, the patient usually within 7 to 11 days after starting symptoms progressive development to coma and decrease, die because of common respiratory failure.

Diagnosis is made by meeting one or more of the following laboratory criteria:

A. Presence of viral antigens;

B. Virus isolated from cell culture or experimental animal inoculation;

C. Virus-specific antibodies are present in cerebrospinal fluid or serum of unvaccinated persons;

D. Detection of viral nucleic acids in living or autopsy samples (such as brain biopsy samples, skin, saliva, concentrated urine) by molecular biological methods.

WHO rabies cases are classified as follows:

(1) Suspected cases: cases that meet the clinical case definition;

(2) Probable cases: suspected cases with a reliable his tory of contact with suspected rabies animals;

(3) Confirmed case: laboratory-confirmed suspected or probable case.

In the absence of an animal exposure history or clinically suspected encephalitis symptoms, a definitive diagnosis can be made if laboratory diagnostic tests are clear.

\section{Definition and Classification of Exposure}

Suspected exposure to rabies virus includes saliva or tissues of animals infected with virus comes into close contact with human damaged skin or mucous membrane by biting, scratching, or licking. For rare cases, rabies can be contracted through organ transplantation or inhalation of aerosols.

Exposure to the rabies virus was classified into three levels according to the nature and severity of the exposure. $^{7-10}$ The categories of exposure and its postexposure prophylaxis (PEP) was grouped as follows:

Level I exposure: one of the following:

1. Touching or feeding animals;

2. Intact skin is licked;

3. Intact skin contact with the secretion or excrement of rabies animals or human rabies cases.

For level I exposure, washing of exposed skin surfaces is recommended by WHO but PEP not indicated.

Level II exposure: one of the following:

1. Nibbling on bare skin;

2. Minor scratches or abrasions without bleeding.

For Level II exposure, the wound should be washed, and the exposed person should be vaccinated immediately.

To identify the levels of exposure: First, observe the skin at the exposed site with the naked eyes. When it is difficult to judge by the naked eye, alcohol can be used to wipe the exposed area. If there is pain, it indicates that there is skin damage (this method is only suitable for testing at the time of injury).

Level III exposure: one of the following:

1. Single or multiple transdermal bites or scratches through the skin;

2. The damaged skin is licked (should pay attention to the skin chapped, scratched and other causes of small skin damage);

3. Mucous membrane is contaminated by animal saliva (such as being licked);

4. Exposure due to direct contact with bats (postexposure prophylaxis should be considered when human-to-bat contact occurs, unless the exposed person rules out bite, scratch or mucous membrane exposure).

\section{Statistical Analysis}

The data were double inputted by Epidata 3.0 to ensure accuracy. After ruling out logical errors, data analysis has 
been initiated in SPSS13.0. One-way chi-square tests and multi-factors logistical regressions have been performed to test the association between the exposure features and the length of the incubation period. A P value less than 0.05 is considered statistically significant.

\section{Results}

\section{Exposure and Rabies Incidence}

From 2013 to 2018, a total of 2,028,691 exposed individuals were received in the outpatient department of rabies exposure in Hubei province, including 314,468 cases in 2013, 250,348 cases in $2014,331,435$ cases in $2015,276,579$ cases in 2016, 438,521 cases in 2017 and 417,340 cases in 2018 (Figure 1). Among those cases, Dog bites were the most common, accounting for $78.61 \%$. Cat bites accounted for $15.35 \%$; Bites from other animals accounted for $6.04 \%$ (Figure 2). Among those exposed cases, 221 rabies cases were confirmed and deceased in Hubei province.

\section{The Demographic Distribution of Rabies in Central China}

The reported incidence of rabies remains constant from 2013 to 2018, including 39 cases reported in 2013, 39 in 2014, 37 in 2015, 31 in 2016, 39 in 2017, and 36 in 2018. Cases were reported throughout the year (Figure 3). In addition to the Snj area, cases have been reported in other areas of the province, among which the top five reported cases are Xy city (43 cases), Yc city (40 cases), Jz city (31 cases), Sy city
(21 cases) and Jm city (21 cases) (Figure 4). Among the 221 rabies cases, the age distribution of infected patients ranged from 2 years to 93 years. Cases are mostly reported in the following three age groups: $60-65$ age group (38 cases), 50 55 age group (35 cases), 55-60 age group (30 cases). $76.02 \%$ of cases were aged over 50 (Figure 5). Among all occupations, farmers had the largest incidence (169 cases, accounting for $76.47 \%$ ), followed by housework (21 cases, $9.50 \%$ ) (Figure 6). The male-to-female ratio of rabies infection in Hubei was 1.91 to 1.

\section{Epidemiological Analysis of Rabies Cases}

A total of 164 cases were applied to the final analysis compared to 221 targeted cases. Missing data on disease risk exposure and disposal may be related to the high case fatality rate of rabies.

\section{The Source of Infection}

Of the 164 cases with known histories of exposure, 148 (90.24\%) were attributed to dogs, 11 by cats, 2 by other animals and 3 by both dogs and cats (difficult to determine whether dog or cat is responsible for the human rabies case). Among the cases only attributed to dogs, 84 (56.75\%) of which were exposed to the dogs of their own or neighbors, and $64(43.25 \%)$ were exposed to stray dogs. For cases of exposure to the dogs of their own, hosts reported that their dogs look healthy in appearance while biting. Among those cases, 12 animals survived; none of them had a history of immunization (Table 1).

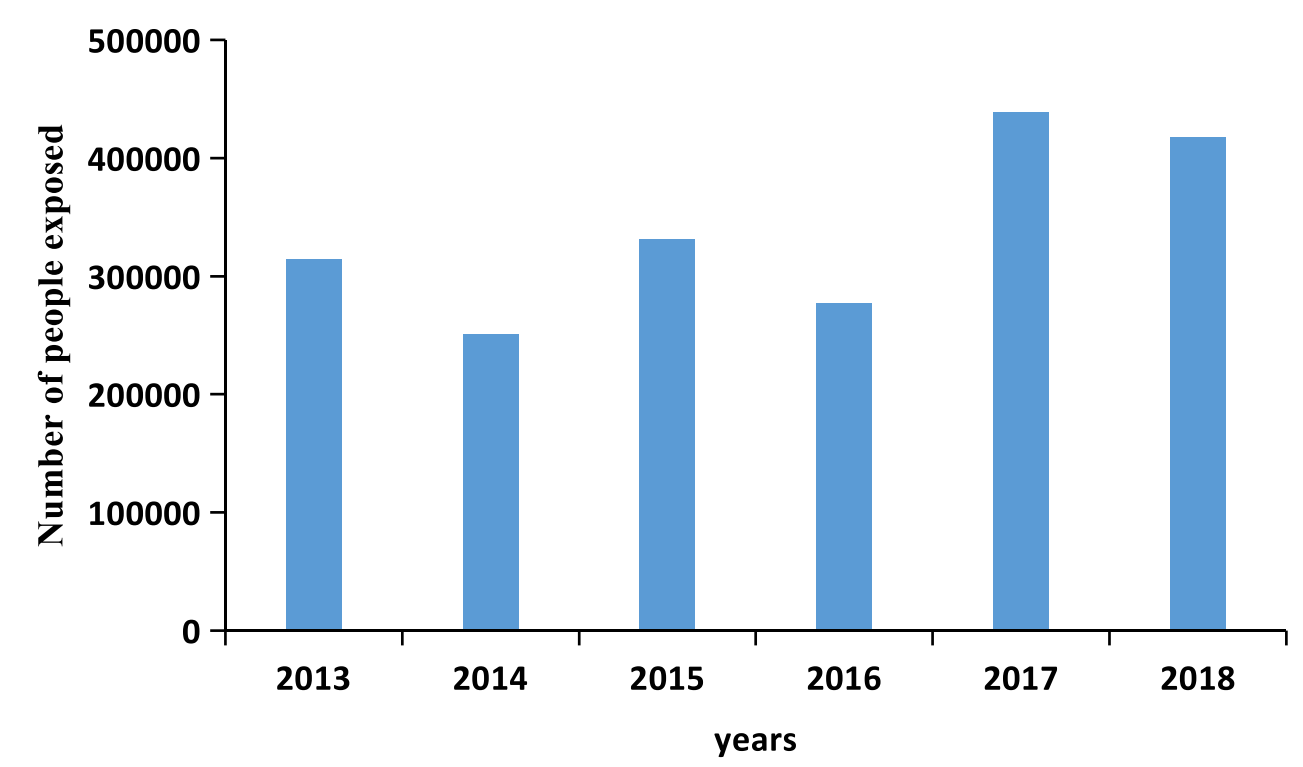

Figure I Number of people exposed to animals in Central China from 2013 to 2018. 


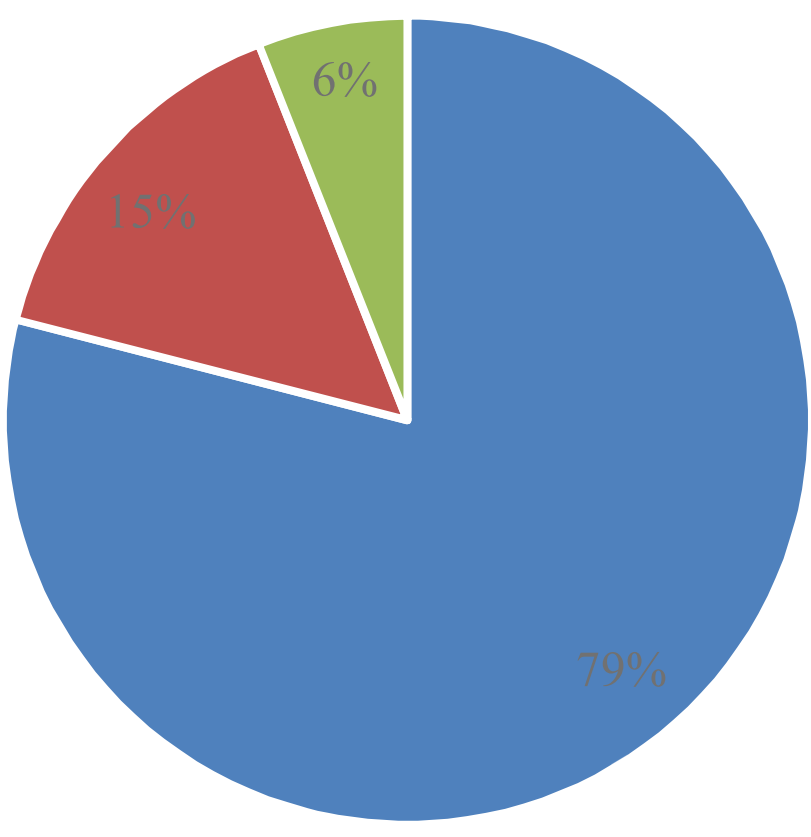

- Dogs - Cats - Other animals

Figure 2 The ratio of bites in Central China from 2013 to 2018.

\section{Site and Severity of Exposure}

The anatomical sites of exposure were the head, face, and neck in 19 cases $(11.59 \%)$, the hands in 70 cases $(42.68 \%)$, the arms in 22 cases $(13.41 \%)$, the trunk in 3 cases $(1.83 \%)$, and lower limbs in 64 cases $(39.02 \%)$. There is a case of exposure of face and head for a child under 10 years old. Among those cases, 7 cases (4.27\%) were level I exposure, 50 cases $(30.49 \%)$ were level II exposure, while 104 cases (63.41\%) were level III exposure. For 3 cases (1.83\%), exposure levels are unknown.

\section{The Wound Management and Immune Status}

Of the 164 patients, 55 (33.54\%) received wound care, including 11 treated by medical institutions and 44 by themselves. 12 patients (11 with level III exposure and 1 with level I exposure) received wound care and were vaccinated. 6 patients were injected with immunoglobulin. A total of 109 cases $(66.46 \%)$ neither received wound care nor vaccinated.

\section{The Incubation Period of Cases}

Among the 164 patients, the incubation period ranged from 3 days to 18,406 days (about 50 years), with a median incubation period of 71 days. The incubation period was less than 1 week in 3 cases (1.83\%), above 1 week but less than 1 month in 31 patients (18.90\%), 1 month to 3 months in 46 patients $(28.05 \%)$. A total of 127 patients $(77.44 \%)$ have incubation periods of less than one year. 16 cases $(9.76 \%)$ had an incubation period of more than 1 year, and 21 cases $(12.80 \%)$ had an unknown incubation period.

1. There was no statistically significant difference in the incubation period of rabies cases with different genders, ages and occupations (Table 2).

2. There were statistically significant differences in the length of the incubation period of rabies cases at different exposed sites, but no statistically

\section{3-2018Year Average 2016 Year 2017 Year $\longrightarrow 2018$ Year}

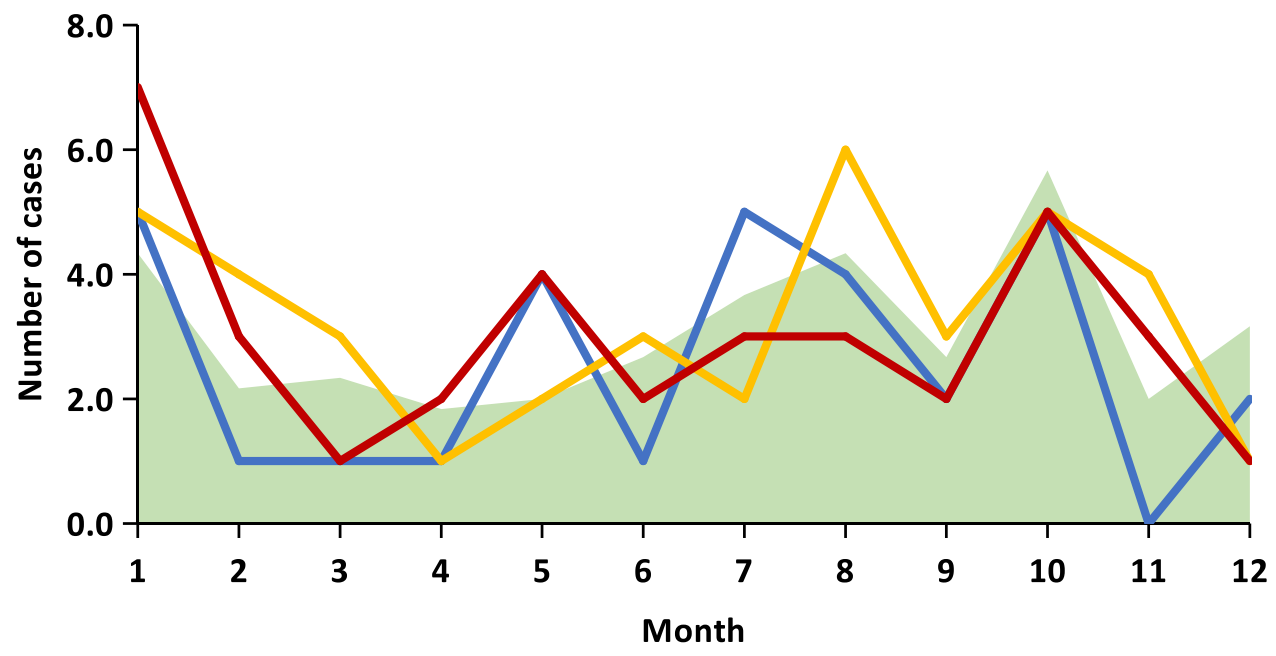

Figure 3 Incidence trend of rabies in different months from 2016 to 2018 in Central China. 


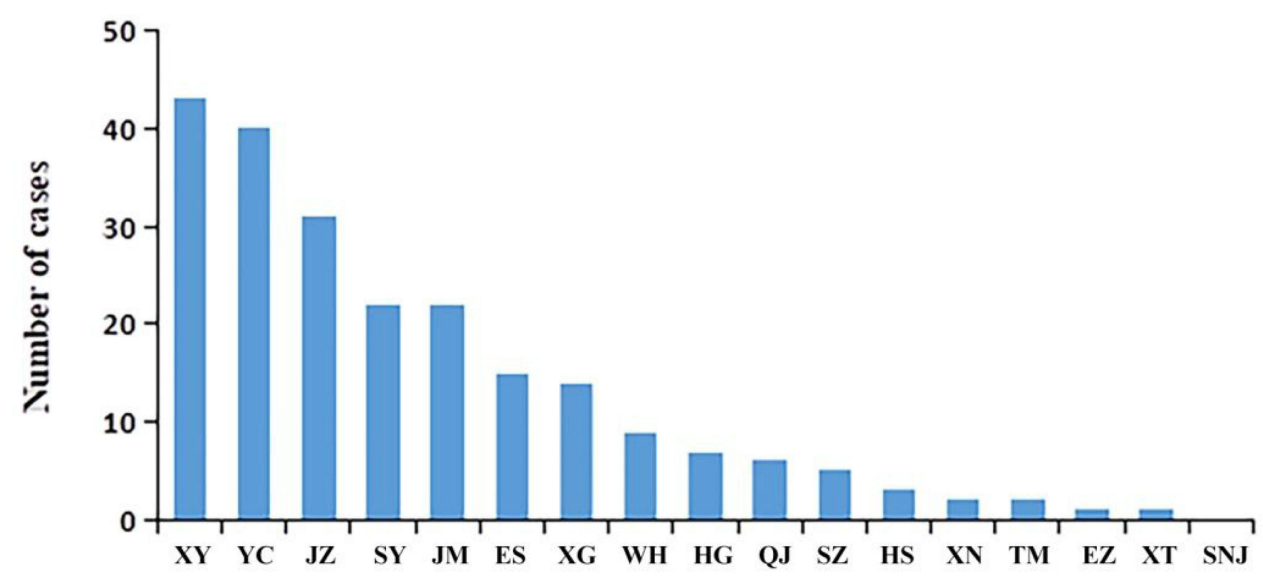

Figure 4 Statistics of rabies incidence in various regions of Central China from 2013 to.

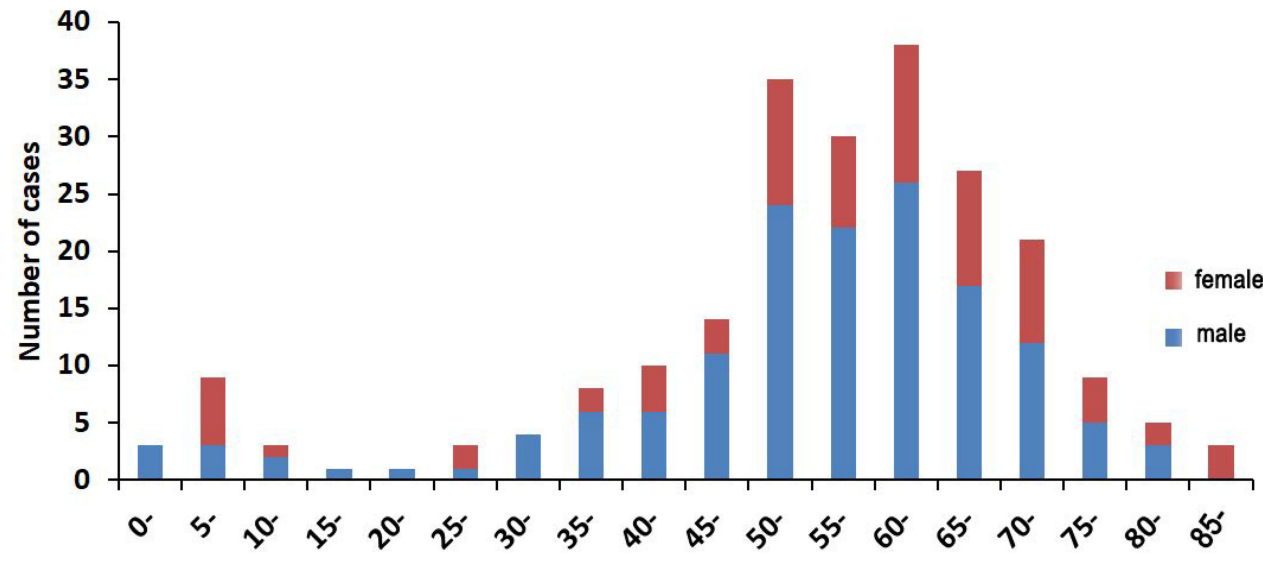

Figure 5 Distribution of rabies in different age groups in Central China from 2013 to 2018.

significant differences in the incubation period of rabies cases with varying modes and degrees of exposure. Specifically, patients whose head, necks, and torsos were exposed have significantly shorter incubation periods than those with other sites exposed (Table 3).

3. There was no statistically significant difference in the incubation periods of rabies cases among species of hurtful animals, sources of injury animals, causes of animal injuries, and wound animal outcomes (Table 1).

4. There was a statistically significant difference in the length of the incubation period between wound treated and untreated rabies cases after exposure. The incubation period of the rabies cases with irrigation was statistically significantly different from that of cases without irrigation $(\mathrm{p}<0.05)$. The average incubation period of the irrigated rabies cases was shorter than that of the untreated. However, there was no statistically significant difference in the incubation period of cases with or without disinfection and suture (Table 4).

5. The incubation periods of the vaccinated patients are statistically significantly shorter than that of the unvaccinated patients (Table 5).

\section{Discussion}

The data analysis results in the recent five years showed that the trend of reported cases of rabies in Hubei Province was flat, and there were no regional outbreaks or largescale outbreaks. Admittedly, due to the increase in the autotrophic pet population, ${ }^{11-15}$ the number of animal exposures in the past five years has generally shown an increasing trend, which brings challenges to the prevention and control of rabies. However, the incidence of rabies has 


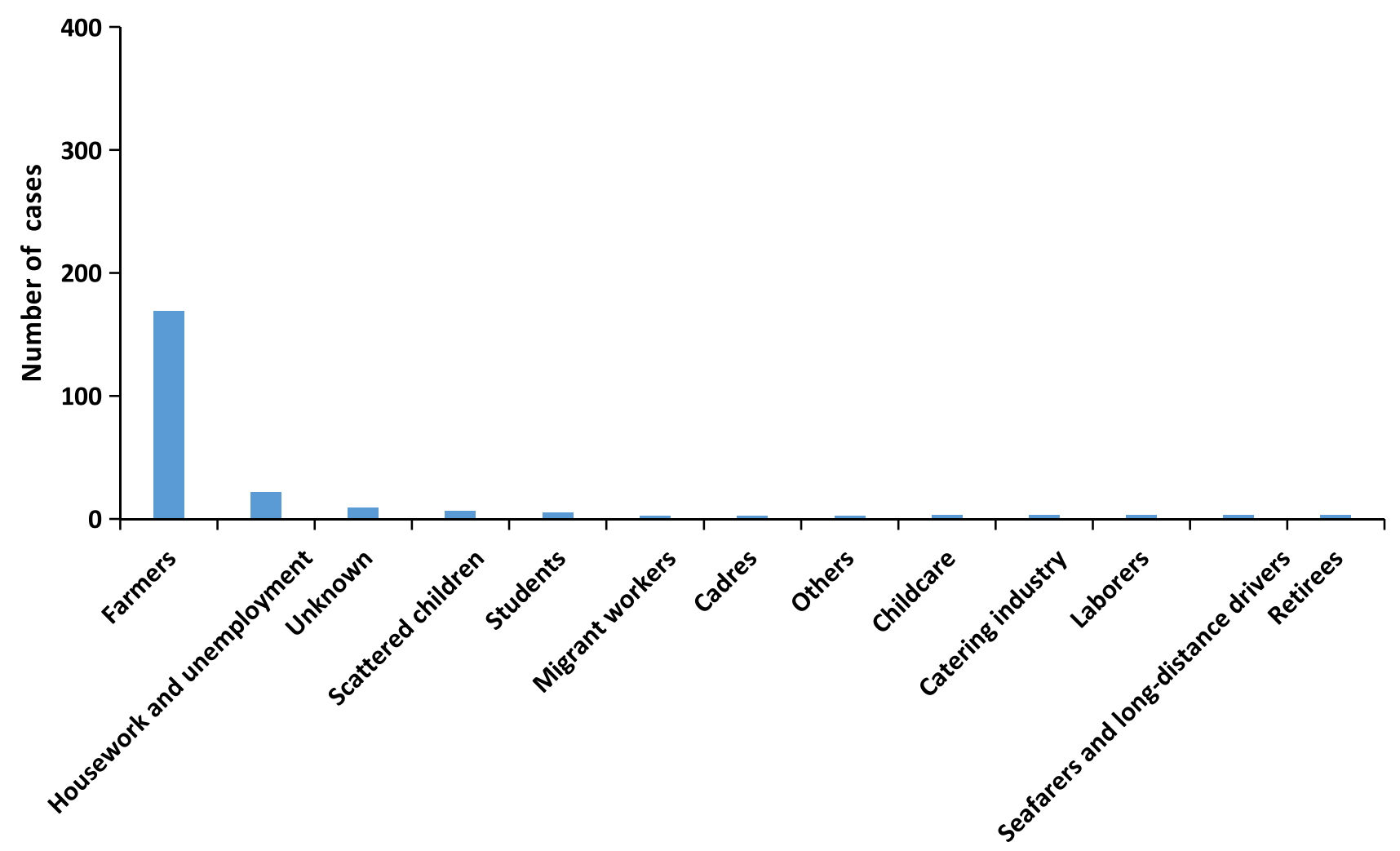

Figure 6 Occupational distribution of rabies in Central China from 2013 to 2018.

remained stable in a relatively small range during five years. In the research process, it is also necessary to rule out confounding and bias, such as the insensitivity of epidemic surveillance.

Among those investigated cases, the male-to-female ratio is greater than 1 , which is consistent with past studies on the distribution of rabies cases. Farmers constitute the majority of the cases, which is in accordance with the general occupational distribution of rabies in China and other countries. ${ }^{16-19} 76.02 \%$ of patients whose age of onset is older than 50. Notably, in China, the age of farmers is usually higher than 50 years old, which makes the occupational distribution of rabies coincide with the age distribution. Possible explanations include first, working in the field makes the risk exposure time longer (more likely to be in contact with wild animals); second, the patient usually lacks knowledge of rabies and will not seek treatment after exposure; third, improper case management and limited PEP resources in rural areas; fourth, the inaccessibility and unaffordable ability of medical services. ${ }^{20-24}$ In summary, in Central China, rural areas still have a higher incidence of rabies than urban areas. The target population for rabies prevention and control should be the rural middle-aged working population.
The duration of the incubation period in the study group ranged from 3 days to 18,406 days. $49.39 \%$ of cases onset were within 3 months, and $77.44 \%$ of cases onset were within 1 year. Notably, the most prolonged incubation period among the reported cases exceeded 50 years, which was consistent with the data reported in relevant literature. ${ }^{25-28}$ In general, the confirmed cases are mainly the second and third levels of exposure. The proportion of wound treatment, irrigation, disinfection, suturing, and vaccination among the exposed population is still low. Only 12 cases were vaccinated after exposure (7.32\%), and only 6 cases $(3.66 \%)$ followed with the administration of rabies immunoglobulin. Admittedly, compared with other developing countries in Asia and Africa, rabies vaccine and Rabies Immune Globulin (RIG) are widely available in China, but the coverage of receiving post-exposure prophylaxis among exposed cases in Central China is still very low. ${ }^{29}$ The reason may be traced back to the fact that people do not have a comprehensive understanding of rabies and its standard post-exposure prophylaxis. Notably, in some cases, even vaccinated, patients' compliance with the recommended dosing schedule of vaccines is still challenging. ${ }^{30-32}$ The average time from the onset of symptoms to decrease 
Table I Factors of Injury and Incubation Period in Rabies Cases

\begin{tabular}{|c|c|c|c|c|c|}
\hline Group & Number & Percent (\%) & Median (Quartile Spacing) & $\chi^{2}$ & $p$ \\
\hline \multicolumn{6}{|c|}{ Species of hurtful animals } \\
\hline Dogs & 148 & 90.24 & $71.0(32.00 \sim 168.00)$ & 2.733 & 0.255 \\
\hline Cats & 11 & 6.71 & $54.0(35.00 \sim 119.50)$ & & \\
\hline Others & 5 & 3.05 & 3876.5 (I25.00 7628.00) & & \\
\hline \multicolumn{6}{|c|}{ Source of hurtful animals } \\
\hline Autotrophic animals & 58 & 35.37 & $69.0(35.25 \sim 160.25)$ & 2.781 & 0.427 \\
\hline Adjacent animals & 26 & 15.85 & $35.0(16.50 \sim 121.50)$ & & \\
\hline Stray animals & 65 & 39.63 & $75.0(30.75 \sim 187.75)$ & & \\
\hline Wild animals & 7 & 4.27 & $115.5(106.00 \sim 125.00)$ & & \\
\hline \multicolumn{6}{|c|}{ Causes of animal injury } \\
\hline Active attack & 98 & 59.76 & $73.0(33.50 \sim 190.50)$ & 1.235 & 0.745 \\
\hline Self-defense wound & 15 & 9.15 & $64.0(25.50 \sim 175.75)$ & & \\
\hline Teasing & 25 & 15.24 & $88.5(32.25 \sim 142.25)$ & & \\
\hline Others or unknown & 18 & 10.98 & $41.0(28.00 \sim 131.00)$ & & \\
\hline \multicolumn{6}{|c|}{ Hurtful animals outcome } \\
\hline Normal & 12 & 7.32 & I I 2.0 (25.50 2739.50) & 7.727 & 0.102 \\
\hline Animals killed & 61 & 37.20 & $49.5(25.00-107.00)$ & & \\
\hline Missing & 60 & 36.59 & $75.0(33.50-202.00)$ & & \\
\hline Paroxysm & 9 & 5.49 & $93.0(34.75 \sim 195.50)$ & & \\
\hline Other & 24 & 14.63 & $125.0(45.00 \sim 298.00)$ & & \\
\hline
\end{tabular}

ranged from 7 to 11 days. The majority of fatal cases died of respiratory failure and Neuronal dysfunction. ${ }^{33-36}$ In view of the current status of the rabies epidemic in Central China, the popularization of rabies knowledge should be enhanced, especially in the rural region. At the same time, the standardization, accessibility, and affordability of rabies PEP should be further enhanced to reduce further death risk.

Rabies is still a challenge to the population health of Central China, especially in rural areas. In Hubei, new cases of rabies are reported nearly every month. The incubation period, ranging from 3 days to 50 years, was found to be

Table 2 The General Demography and Incubation Period of Rabies Cases

\begin{tabular}{|c|c|c|c|c|}
\hline Group & Number & Percent (\%) & Median (Quartile Spacing) & Statistical Test \\
\hline \multicolumn{5}{|l|}{ Gender } \\
\hline Male & 104 & 63.41 & $68.0(30.00 \sim 147.50)$ & $Z=-0.622, p=0.534$ \\
\hline Female & 60 & 36.59 & $82.0(33.00 \sim 183.50)$ & \\
\hline \multicolumn{5}{|l|}{ Age } \\
\hline$\leq 6$ & 7 & 4.27 & $15.0(10.25 \sim 30.25)$ & $\chi^{2}=7.677, p=0.104$ \\
\hline $7 \sim 17$ & 5 & 3.05 & 71.5 (35.50 92.50) & \\
\hline $18 \sim 40$ & 15 & 9.15 & $36.0(21.00 \sim 232.00)$ & \\
\hline $41 \sim 65$ & 66 & 40.24 & $77.0(30.00 \sim 218.00)$ & \\
\hline$\geq 66$ & 71 & 43.29 & $74.0(41.50 \sim 154.00)$ & \\
\hline \multicolumn{5}{|c|}{ Occupation } \\
\hline Peasant & 135 & 82.32 & $77.0(33.00 \sim 164.00)$ & $Z=-1.240, p=0.215$ \\
\hline Other & 29 & 17.68 & $44.0(20.50 \sim 196.25)$ & \\
\hline
\end{tabular}


Table 3 The Association Between Exposure and Incubation Period

\begin{tabular}{|c|c|c|c|c|c|}
\hline Group & Number & Percent (\%) & Median (Quartile Spacing) & $\chi^{2}$ & $p$ \\
\hline \multicolumn{6}{|l|}{ Mode of exposure } \\
\hline Bite & 145 & 88.41 & $73.0(32.00 \sim 166.00)$ & 0.636 & 0.727 \\
\hline Scratch & 15 & 9.15 & $44.5(30.25 \sim 189.00)$ & & \\
\hline Other & 4 & 2.44 & $79.0(15.00 \sim 143.00)$ & & \\
\hline \multicolumn{6}{|l|}{ Degree of exposure } \\
\hline Level I & 7 & 4.27 & $83.5(31.00 \sim 698.00)$ & 0.503 & 0.778 \\
\hline Level II & 50 & 30.49 & $70.5(31.25 \sim 202.25)$ & & \\
\hline Level III & 104 & 63.41 & $73.5(32.00 \sim 162.75)$ & & \\
\hline \multicolumn{6}{|l|}{ Exposed area } \\
\hline Head, neck and torso & 19 & 11.59 & $30.0(16.00 \sim 77.50)$ & 11.674 & 0.02 \\
\hline Arm & 22 & $|3.4|$ & $75.0(28.00 \sim 129.50)$ & & \\
\hline Hand & 70 & 42.68 & $70.0(32.00 \sim 149.00)$ & & \\
\hline Above the knee & 12 & 7.32 & $218.0(86.50 \sim 2660.25)$ & & \\
\hline Below the knee & 52 & 31.71 & $72.5(32.25 \sim 205.00)$ & & \\
\hline
\end{tabular}

Table 4 Treatment of Wounds and Incubation Period After Exposure in 164 Rabies Cases

\begin{tabular}{|c|c|c|c|c|}
\hline Group & Number & Percent(\%) & Median (Quartile Spacing) & Statistical Test \\
\hline \multicolumn{5}{|l|}{ Wound management } \\
\hline Untreated & III & 67.68 & $88.0(36.00 \sim 208.50)$ & $\chi^{2}=16.142, p=0.000$ \\
\hline Self treatment & 44 & 26.83 & $64.0(30.50 \sim 116.00)$ & \\
\hline Medical institution treatment & 11 & 6.71 & $16.0(14.00 \sim 19.00)$ & \\
\hline \multicolumn{5}{|l|}{ Irrigation } \\
\hline Yes & 54 & 32.93 & $49.5(18.00 \sim 103.25)$ & $Z=-2.873, p=0.004$ \\
\hline No & 110 & 67.07 & $89.0(36.00 \sim 208.00)$ & \\
\hline \multicolumn{5}{|l|}{ Disinfection } \\
\hline Yes & 24 & 14.63 & $60.0(16.00 \sim 168.00)$ & $Z=-1.530, p=0.126$ \\
\hline No & 140 & 85.37 & $74.0(34.00 \sim 162.75)$ & \\
\hline \multicolumn{5}{|l|}{ Suture } \\
\hline Yes & 7 & 4.27 & $19.0(14.00 \sim 168.00)$ & $Z=-1.825, p=0.068$ \\
\hline No & 146 & 93.59 & $73(33.0 \sim 174.5)$ & \\
\hline
\end{tabular}

statistically significant with the sites of exposure. In other words, patients whose head, necks, and torsos exposed have significantly shorter incubation periods than that of the other. Even though the vaccine has been used for rabies postintervention in China since 1981, this paper found that the overall coverage of standard PEP among vulnerable groups in Central China is still low. With regard to gender and age distribution, middle-aged men have a higher risk exposure ratio. To investigate the potential causes should explore the social and economic factors embedded.
Following the above epidemiological study, the paper suggested a list of measures to be taken in a targeted manner: From the animal side, on the one hand, strict control and immunization on stray animals should be stressed. On the other hand, hosts should take their pet animals to visit veterinarians on a regular basis and keep rabies vaccinations up-to-date. From the human side, strict measures must be enhanced to ensure the availability, accessibility and affordability of Pre-exposure prophylaxis (PrEP) and PEP. For instance, standard PEP training for rural medical staff should 
Table 5 Inoculation and Incubation Period of Rabies Cases

\begin{tabular}{|l|c|c|c|c|}
\hline Group & Number & $\begin{array}{c}\text { Percent } \\
\text { (\%) }\end{array}$ & $\begin{array}{c}\text { Median } \\
\text { (Quartile } \\
\text { Spacing) }\end{array}$ & $\begin{array}{c}\text { Statistical } \\
\text { Test }\end{array}$ \\
No & 152 & 92.68 & $\begin{array}{c}75.0 \\
(34.00 \sim 183.00)\end{array}$ & $\begin{array}{c}\mathrm{Z}=-3.131, \\
\mathrm{~T}=0.002\end{array}$ \\
\hline Vaccination & 12 & 7.32 & $60.25)$ \\
\hline
\end{tabular}

be strengthened. It is also urgent to enhance the local monitoring system of vaccine compliance.

\section{Ethical Approval}

The study protocol and the questionnaire were reviewed and approved by the Research Ethics Committee in Hubei Center for Disease Control and Prevention, 430,079, Wuhan, China. Participation was voluntary with informed consent forms signed before participation. The data could be accessed from Prof. Yeqing Tong.

\section{Acknowledgments}

This study was supported by Hubei Young Talent Plan (2017) and Hubei Medical Youth Reserve Talent program (2019) as well as Hubei Technological Innovation Plan (2017ADC061) as well as Hubei Outstanding Medical Academic Leader Program (20131016). We must say "thank you" to all participants of this study and Katherine A Mason for her language help.

\section{Disclosure}

The authors declare no conflicts of interest.

\section{References}

1. Cavalcante KKS, Florencio C, Moreno JO, Correia FGS, Alencar CH. Post-exposure human rabies prophylaxis: spatial patterns of inadequate procedures in Ceara - Brazil, 2007 to 2015. Rev Soc Bras Med Trop. 2019;53:e20190247. doi:10.1590/0037-8682-0247-2019

2. Du Z, Chen Q, Lyu X, Wang T, Wang C. Analyzing the distribution of rabies clinics and achievements of standardized rabies clinics implementation in mainland China. BMC Health Serv Res. 2019;19:955. doi:10.1186/s12913-019-4730-9

3. Manoharan A, Chellaiyan VG, Madhusudan M. Effect of educational intervention on the knowledge of rabies among medical school students of Chennai. J Educ Health Promot. 2019;8:208, 161_19.

4. Duarte NF, Alencar CH, Cavalcante KK, et al. Increased detection of rabies virus in bats in Ceara State (Northeast Brazil) after implementation of a passive surveillance programme. Zoonoses Public Health. 2020;67(2):186-192.
5. Kiffner C, Latzer M, Vise R, et al. Comparative knowledge, attitudes, and practices regarding anthrax, brucellosis, and rabies in three districts of northern Tanzania. BMC Public Health. 2019;19(1):1625. doi:10.1186/s12889-019-7900-0

6. Kimball BA, Volker SF, Griffin DL, Johnson SR, Gilbert AT, Recuenco S. Volatile metabolomic signatures of rabies immunization in two mesocarnivore species. PLoS Negl Trop Dis. 2019;13(12): e0007911. doi:10.1371/journal.pntd.0007911

7. WHO. WHO Expert Consultation on Rabies. [R]. Geneva: WHO;2013. Contract No.: 982.

8. Manning SE, Rupprecht CE, Fishbein D, et al. Human rabies prevention--United States, 2008: recommendations of the advisory committee on immunization practices. MMWR Recomm Rep. 2008;57 (RR-3):1-28.68.

9. World Health Organization. Announcing the publication of the WHO immunological basis for immunization series module on pertussis vaccines. Vaccine. 2019 Jan 7;37(2):217-218. doi:10.1016/j. vaccine.2017.10.110.

10. Publication W. Rabies vaccines: WHO position paper-recommendations. Vaccine. 2010;28(44):7140. doi:10.1016/j. vaccine.2010.08.082

11. Endy TP, Keiser PB, Cibula D, et al. Effect of antimalarial drugs on the immune response to intramuscular rabies vaccination using a postexposure prophylaxis regimen. J Infect Dis. 2019.

12. Bouli F, Awah-Ndukum J, Mingoas KJ-P, Tejiokem MC, Tchoumboue J. Dog demographics and husbandry practices related with rabies in Cameroon. Trop Anim Health Prod. 2020;52 (3):979-987. doi:10.1007/s11250-019-02085-9

13. Barcenas-Reyes I, Nieves-Martínez DP, Cuador-Gil JQ, et al. Spatiotemporal analysis of rabies in cattle in central Mexico. Geospat Health. 2019;14(2). doi:10.4081/gh.2019.805

14. Williams VF, Taubman SB, Stahlman S. Animal bites and rabies post-exposure prophylaxis, active and reserve components, U.S. armed forces, 2011-2018. Msmr. 2019;26:13-20.

15. Reece J. Working together within the profession to eradicate rabies. Vet Rec. 2019;185(16):513-514. doi:10.1136/vr.16126

16. Maher EK, Ward MP, Brookes VJ. Investigation of the temporal roaming behaviour of free-roaming domestic dogs in Indigenous communities in northern Australia to inform rabies incursion preparedness. Sci Rep. 2019;9(1):14893. doi:10.1038/s41598-01951447-8

17. Sudarshan MK, Ashwath Narayana DH. Background paper for developing a policy for the use of rabies biologicals and vaccination of humans in India. Indian J Public Health. 2019;63:S51S53.

18. Middlemiss C. The UK's contribution to eliminating rabies. Vet Rec. 2019;185:379. doi:10.1136/vr.15695

19. Yoder J, Younce E, Lankester F, Palmer GH. Healthcare demand in response to rabies elimination campaigns in Latin America. PLoS Negl Trop Dis. 2019;13:e007630. doi:10.1371/journal.pntd.000 7630

20. Mbaipago J, Mindekem R, Oussiguere A, et al. Rabies knowledge and practices among human and veterinary health workers in Chad. Acta Trop. 2019;202:105180.

21. O'Sullivan B, Burke R, Bassaline D. Notes from the field: rabies exposures from fox bites and challenges to completing postexposure prophylaxis after hurricane irma - Palm Beach County, Florida, August-September 2017. MMWR Morb Mortal Wkly Rep. 2019;68:795-797. doi:10.15585/mmwr.mm6836a4

22. Coetzer A, Scott TP, Noor K, Gwenhure LF, Nel LH. A novel integrated and labile eHealth system for monitoring dog rabies vaccination Campaigns. Vaccines. 2019;7(3):108. doi:10.3390/vaccines 7030108 
23. Gill G, Singh BB, Dhand NK, et al. Estimation of the incidence of animal rabies in Punjab, India. PLoS One. 2019;14(9):e0222198. doi:10.1371/journal.pone.0222198

24. Khan A, Ayaz R, Mehtab A, et al. Knowledge, attitude \& practices (KAPs) regarding rabies endemicity among the community members, Pakistan. Acta Trop. 2019;200:105156. doi:10.1016/j. actatropica.2019.105156

25. Davis AD, Dupuis M, Rudd RJ. Extended incubation period of rabies virus in a captive big brown bat (Eptesicus fuscus). $J$ Wildl Dis. 2012;48(2):508-511. doi:10.7589/0090-3558-48.2.508

26. Shankar SK, Sapico S, Pinto RGW, et al. Rabies viral encephalitis with proable 25 year incubation period! Ann Indian Acad Neurol. 2012;15(3):221-223. doi:10.4103/0972-2327.99728

27. Tojinbara K, Sugiura K, Yamada A, et al. Estimating the probability distribution of the incubation period for rabies using data from the 1948-1954 rabies epidemic in Tokyo. Prev Vet Med. 2016;123:102-105. doi:10.1016/j.prevetmed.2015.11.018

28. Johnson N, Fooks A, McColl K, et al. Reexamination of human rabies case with long incubation, Australia. Emerg Infect Dis. 2008;14(12):1950-1951. doi:10.3201/eid1412.080944

29. Sreenivasan N, Li A, Shiferaw M, et al. Overview of rabies postexposure prophylaxis access, procurement and distribution in selected countries in Asia and Africa, 2017-2018. Vaccine. 2019;Suppl 37: A6-A13. doi:10.1016/j.vaccine.2019.04.024
30. Mbilo C, Kabongo J-B, Pyana PP, et al. Dog ecology, bite incidence, and disease awareness: a cross-sectional survey among a rabies-affected community in the Democratic Republic of the Congo. Vaccines. 2019;7(3):98. doi:10.3390/vaccines7030098

31. Amanatin A, Sudarnika E, Lukman D, et al. Risk assessment on rabies entry through hunting dog movement with semi-quantitative approach to Sumatera Island, Indonesia. J Adv Vet Anim Res. 2019;6 (2):148-157. doi:10.5455/javar.2019.f325

32. Ntampaka P, Nyaga PN, Niragire F, et al. Knowledge, attitudes and practices regarding rabies and its control among dog owners in Kigali city, Rwanda. PLoS One. 2019;14(8):e0210044. doi:10.1371/journal. pone. 0210044

33. Banyard AC, Tordo N, et al. Rabies pathogenesis and immunology. Rev Sci Tech. 2018;37(2):323-330. doi:10.20506/rst.37.2.2805

34. Cole E, Gillespie S, Vulliamy P, et al. Multiple organ dysfunction after trauma. Br J Surg. 2020;107(4):402-412. doi:10.1002/bjs.11361

35. Ugolini G, Hemachudha T. Rabies: changing prophylaxis and new insights in pathophysiology. Curr Opin Infect Dis. 2018;31 (1):93-101. doi:10.1097/QCO.0000000000000420

36. Fu ZF, Jackson AC. Neuronal dysfunction and death in rabies virus infection. $J$ Neurovirol. 2005;11(1):101-106. doi:10.1080/ 13550280590900445
Infection and Drug Resistance

\section{Publish your work in this journal}

Infection and Drug Resistance is an international, peer-reviewed openaccess journal that focuses on the optimal treatment of infection (bacterial, fungal and viral) and the development and institution of preventive strategies to minimize the development and spread of resistance. The journal is specifically concerned with the epidemiology of

\section{Dovepress}

antibiotic resistance and the mechanisms of resistance development and diffusion in both hospitals and the community. The manuscript management system is completely online and includes a very quick and fair peerreview system, which is all easy to use. Visit http://www.dovepress.com/ testimonials.php to read real quotes from published authors. 